\section{Thermal proximity coaggregation for system-wide profiling of protein complex dynamics in cells}

\author{
Chris Soon Heng Tan,,${ }^{1,2 *}$ Ka Diam Go, ${ }^{3}$ Xavier Bisteau, ${ }^{1}$ Lingyun Dai, ${ }^{3}$ \\ Chern Han Yong, ${ }^{4,5}$ Nayana Prabhu, ${ }^{3}$ Mert Burak Ozturk, ${ }^{1,6}$ Yan Ting Lim, ${ }^{3}$ \\ Lekshmy Sreekumar, ${ }^{3}$ Johan Lengqvist, ${ }^{7}$ Vinay Tergaonkar, ${ }^{1,6,8}$ Philipp Kaldis, ${ }^{1,6}$ \\ Radoslaw M. Sobota, ${ }^{1,2}$ Pär Nordlund ${ }^{3,1,7 *}$
}

Proteins differentially interact with each other across cellular states and conditions, but an efficient proteome-wide strategy to monitor them is lacking. We report the application of thermal proximity coaggregation (TPCA) for high-throughput intracellular monitoring of protein complex dynamics. Significant TPCA signatures observed among well-validated protein-protein interactions correlate positively with interaction stoichiometry and are statistically observable in more than 350 annotated human protein complexes. Using TPCA, we identified many complexes without detectable differential protein expression, including chromatin-associated complexes, modulated in S phase of the cell cycle. Comparison of six cell lines by TPCA revealed cell-specific interactions even in fundamental cellular processes. TPCA constitutes an approach for system-wide studies of protein complexes in nonengineered cells and tissues and might be used to identify protein complexes that are modulated in diseases.

A living cell arises from a myriad of biomolecule interactions occurring in time and space among proteins, nucleic acids, metabolites, and lipids. Central to this intricate biological network are protein complexes that mediate the biochemical processes and the structural organization of the cell. They assemble and dissociate dynamically according to cellular needs and are implicated in many different diseases $(1,2)$.

Large-scale studies using specific cell lines (3-9), complemented by focused efforts, have contributed to a large protein-protein interaction network depicting the plausible cellular wiring and functional organization of the human proteome. However, the conservation of the assembled protein network and identified protein complexes across cell types, physiological states, and diseased conditions is unclear. Methods that permit efficient, system-wide, and hypothesis-free identification of differentiated protein complexes in nonengineered and diseased cells will expedite biological studies.

${ }^{1}$ Institute of Molecular and Cell Biology (IMCB), A*STAR (Agency for Science, Technology and Research), Singapore ${ }^{2}$ Institute of Medical Biology (IMB), A*STAR (Agency for Science, Technology and Research), Singapore. ${ }^{3}$ School of Biological Sciences, Nanyang Technological University, Singapore. ${ }^{4}$ Program in Cancer and Stem Cell Biology, Duke-National University of Singapore (NUS) Medical School, Singapore. ${ }^{5}$ Centre for Computational Biology, Duke-NUS Medical School, Singapore. ${ }^{6}$ Department of Biochemistry, Yong Loo Lin School of Medicine, National University of Singapore, Singapore. 'Department of Oncology-Pathology, Cancer Center Karolinska, Karolinska Institutet, Stockholm, Sweden. ${ }^{8}$ Centre for Cancer Biology (University of South Australia and SA Pathology), Adelaide, Australia.

*Corresponding author. Email: cshtan@imcb.a-star.edu.sg (C.S.H.T.); pnordlund@ntu.edu.sg (P.N.)
Here, we explored the cellular thermal shift assay (CETSA) for the study of protein complex dynamics (10). By using protein mass spectrometry (MS) with multiplexed quantification for CETSA (MS-CETSA or thermal proteome profiling), melting curves are generated for thousands of proteins (11-13). In this work, we analyzed MS-CETSA data generated from samples without exogenous ligands. We validated thermal proximity coaggregation (TPCA) as an approach for systemwide intracellular monitoring of protein complex dynamics.

TPCA is based on the hypothesis that interacting proteins coaggregate upon heat denaturation, leading to similar solubility across different temperatures (Fig. 1A). We first investigated TPCA with the well-characterized Cdk2-cyclin E1 complex, deriving the melting curves using immunoblots for both proteins overexpressed in human embryonic kidney (HEK) 293T cells (fig. S1, A and B). Individually expressed cyclin E1-V5 and Cdk2-HA (hemagglutinin) display distinct melting curves, but they interact and are stabilized with similar melting curves when coexpressed (Fig. 1B and fig. S1A), consistent with the TPCA hypothesis.

To evaluate the generality of TPCA, we obtained the melting curves for 7693 human proteins (table S1) collated from eight MS-CETSA experiments of the same K562 lysate (Fig. 1C). We assembled 111,776 protein-protein interactions annotated in BioGRID (14), InAct (15), and MINT (16) databases occurring among the 7693 proteins (table S2). Using Euclidean distance as an inverse measure of curve similarity, we observed that interacting protein pairs generally have higher curve similarity than all protein pairs $\left(P<2.2 \times 10^{-16}\right.$, one-tailed Mann-Whitney test, Fig. 1D). This similarity is more pronounced for interactions reported by multiple publications (Fig. 1D and fig. S2). We also observed high curve similarity for interactions from two recent large-scale studies $(5,6)$ using yeast twohybrid $(\mathrm{Y} 2 \mathrm{H})$ and affinity purification (AP)$\mathrm{MS} / \mathrm{MS}$, and for subunit pairs of complexes in the CORUM database (Fig. 1E) (17). Thus, it is unlikely that the observed TPCA signatures arose from ascertainment bias and the experimental methods used.

We computed the average curve similarity among all subunit pairs of each protein complex and assessed TPCA signatures at the protein complex level. For the 558 nonredundant human complexes having at least three subunits with melting curves, 160 exhibited nonrandom TPCA signatures among subunits collectively $(P<0.05$, table S3, Fig. 1F, and fig. S3). Next, we obtained MS-CETSA data from the K562 cell lysate that was depleted of low-molecular weight (LMW) ligands by desalting (table S4). We observed decreased average curve similarity for most protein complexes ( $88 \%$, fig. S4A and table S5), suggesting that LMW ligand depletion caused increased complex dissociation. However, we observed multiple protein complexes with negligible changes in TPCA but with melting curves of all subunits shifted similarly, such as the PA700 proteasome subcomplex that could be due to adenosine $5^{\prime}$-triphosphate (ATP) depletion (Fig. 2A and fig. S5). Thus, whole-protein complexes can remain intact yet thermally destabilized by LMW ligand depletion. In comparison, MS-CETSA data from a new batch of K562 lysate shared good reproducibility with the previous lysate (Pearson's $R=$ 0.88 , fig. S3B). We observed a higher reproducibility (fig. S4C) for proteins with at least three quantified peptides in each data set, and thus combined existing and new data sets for subsequent analysis (table S6).

Six MS-CETSA experiments were performed on intact K562 cells (table S7). We observed melting curves of all protein pairs to be statistically more similar in the lysate data (Fig. 2B), whereas those for interacting protein pairs are statistically more similar in intact cell data (Fig. 2B). We also observed that protein complexes have better average curve similarity among subunits in intact cell data than in lysate data (figs. S6, A to $\mathrm{D}$, and S7). Overall, intact cell data reveal more protein complexes with nonrandom TPCA signatures than the lysate data (Fig. 2C; fig. S6, E and F; and table S8).

TPCA-significant protein complexes in stress response, cell cycle, and DNA processing pathways are statistically enriched in intact cell data over lysate data (fig. S6G). Many DNA-chromatinassociated complexes and membrane-associated complexes-as exemplified by the Nup 107-160 nuclear pore subcomplex and the origin recognition complex, respectively (Fig. 2D)-exhibit significant TPCA signatures only in intact cells, presumably due to disruption of DNA-chromatin and native membranes in cell lysate. Our analysis suggests that the replication factor complex $\mathrm{C}$ (RFC) remains assembled in both intact cell and 
lysate (Fig. 2E, top) but that the whole complex is thermally destabilized in lysate (Fig. 2E, top left), presumably due to the absence of DNAchromatin.
We observed many instances in which subcomplexes exhibit distinct TPCA signatures between intact cell and lysate data sets. For example, the $40 S$ and $60 S$ ribosomal subcom- plexes each have subunits with similar curves that shifted closer to the other subcomplex in intact cell data (Fig. 2F), presumably because more fully assembled ribosomes are translating
A

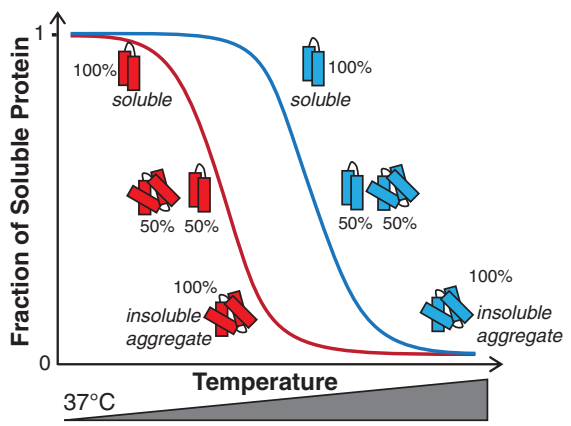

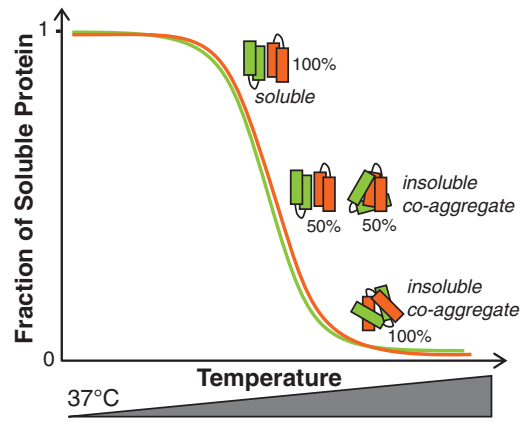

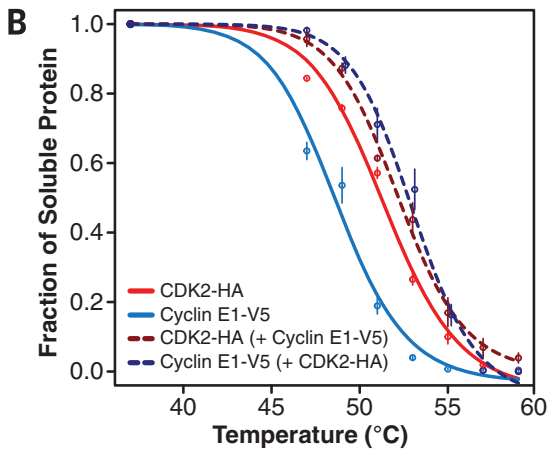

C

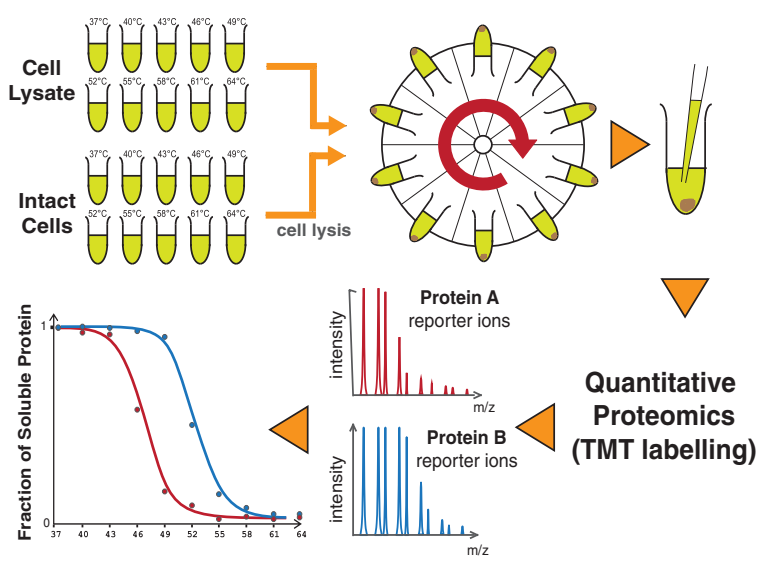

$\mathbf{F}$

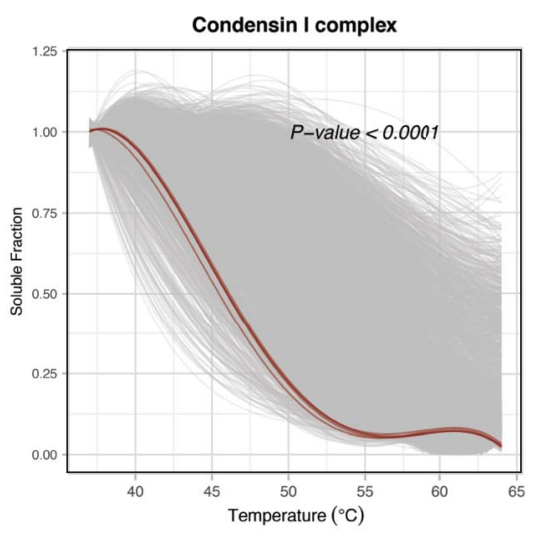

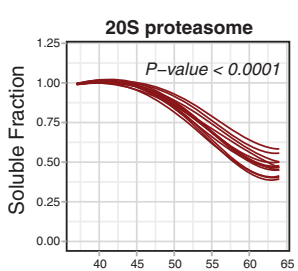

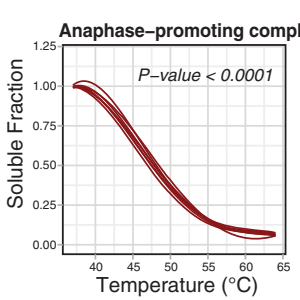

D

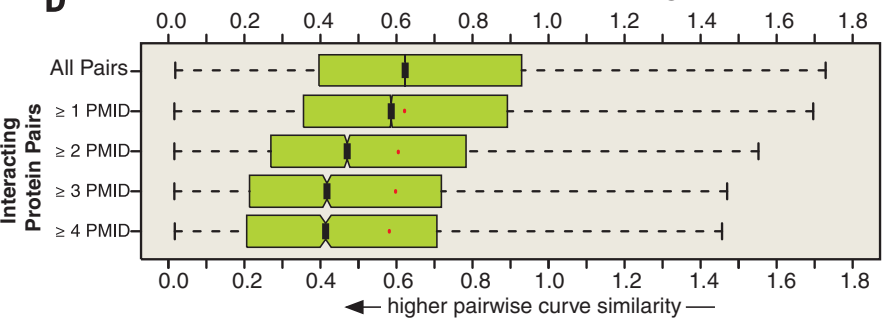

E Distribution of Euclidean Distance Among Protein Pairs

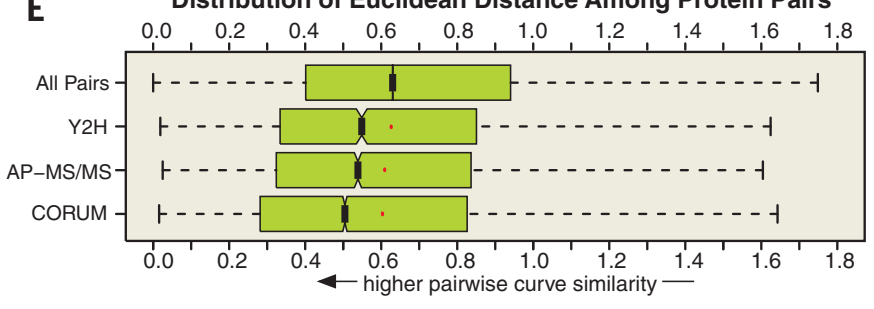

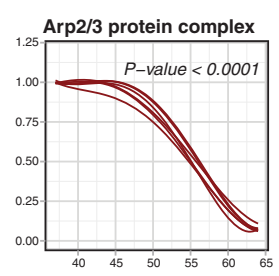

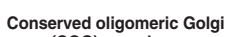

BLOC-1 (biogenesis of lysosome-
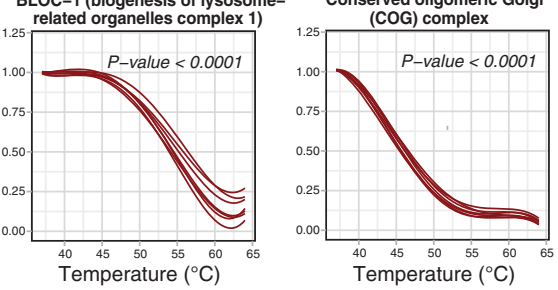

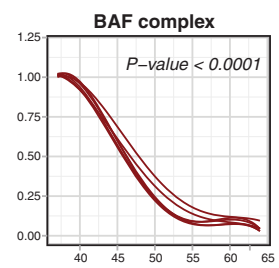

Multisynthetase complex

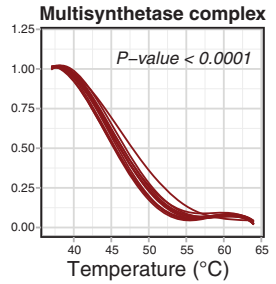

Fig. 1. Interacting protein pairs exhibit strong TPCA signature. (A)

Principle of TPCA for monitoring protein-protein interactions: Coaggregation and precipitation among interacting proteins result in similar protein solubility across different denaturing temperatures. (B) Cointeracting cyclin E1 and Cdk2 exhibit similar melting curves: HEK 293T cells were transfected with plasmids encoding V5-tagged cyclin E1 and/or HA-tagged Cdk2, followed by the intact cell CETSA experiment. Data are from immunoblots of three biological replicates (mean \pm SD) and are fitted with a three-parameter log-logistic function. Representative immunoblots are shown in fig. S1B. (C) Schematic overview of MS-CETSA experiment. m/z, mass/charge ratio; TMT, tandem mass tags. (D) Distribution of melting curve similarities between known interacting protein pairs according to number of reporting publications: PMID, PubMed identifier. Red dots indicate the median of all protein pairs of each respective protein subset. (E) Distribution of melting curve similarities of known interacting protein pairs reported in Rolland et al. (6), Huttlin et al. (5), and CORUM. (F) Protein melting curves of selected protein complexes: red lines, complex subunits. For the leftmost plot, the solubility curves of $\sim 4000$ other proteins are plotted in gray. 
mRNA in cells than in lysate. Another example is the NDC80 kinetochore complex with two distinct subunit groups based on melting curves that are more similar to each other in intact cell data than in lysate data (Fig. 2E, bottom). The two distinct subunit groups correspond to the two stable heterodimers that are associated with each other through a short tetramerization protein region (Fig. 2G) (18, 19). TPCA analysis suggests that the two subcomplexes had dissociated in lysate.
These observations suggest that TPCA can reveal fundamental differences in protein complex organization and their functional states.

The stoichiometry of interaction and abundance between subunits in a complex can potentially
A

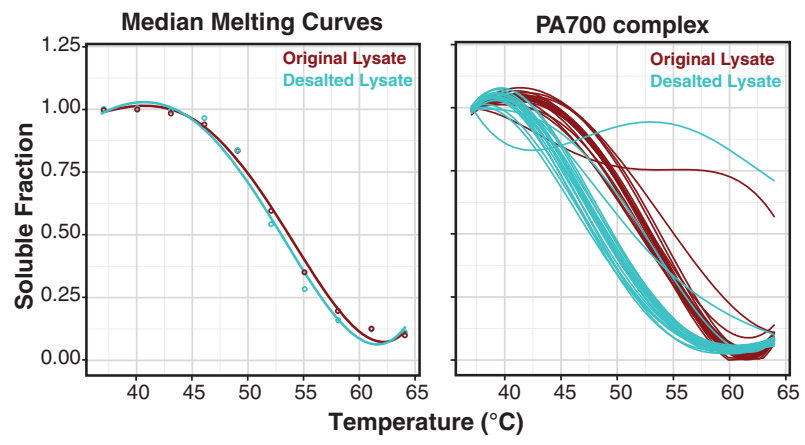

C

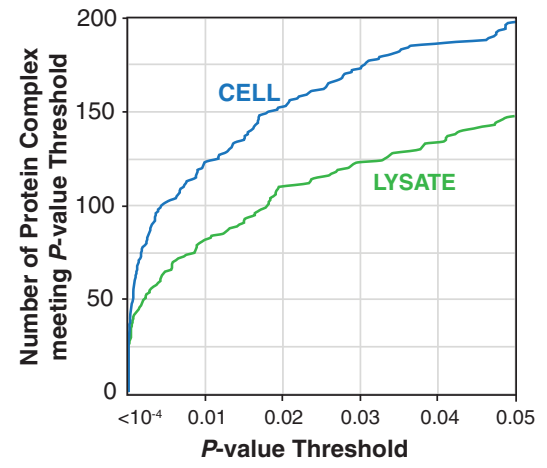

D

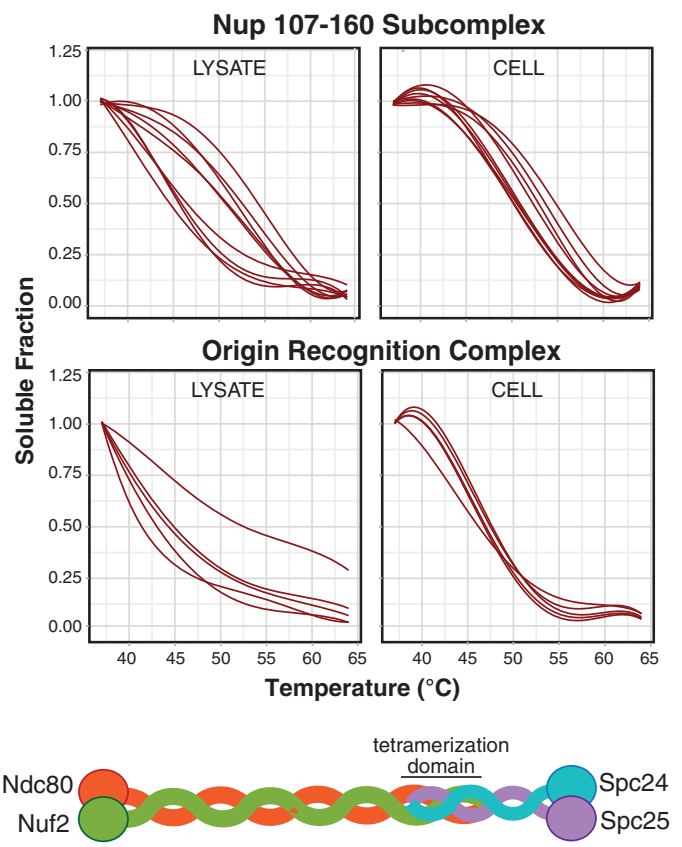

Fig. 2. TPCA signature is stronger in data from intact cells. (A) Effect of desalting cell lysate on subunit melting curves of PA700 proteasome subcomplex. (B) Distribution of melting curve similarities among known interacting protein pairs computed from cell lysate and intact cell data, respectively. Red dots indicate the median of all protein pairs of each

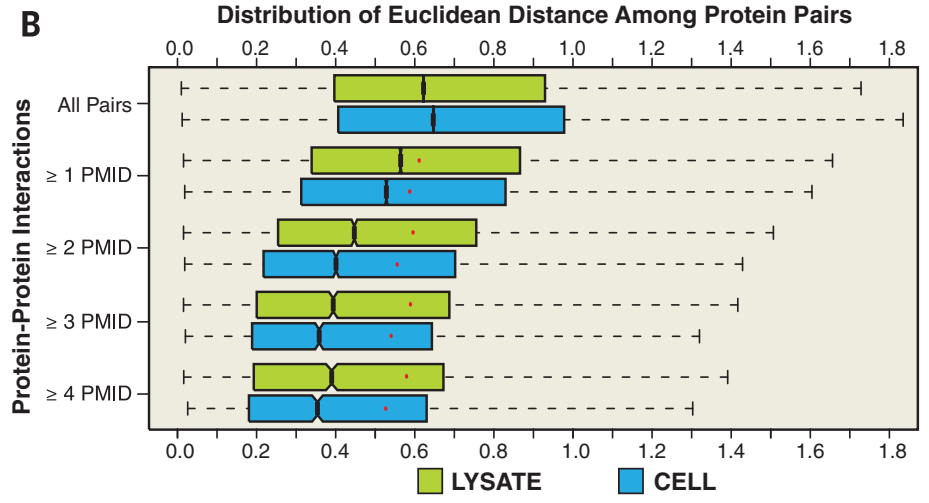

E

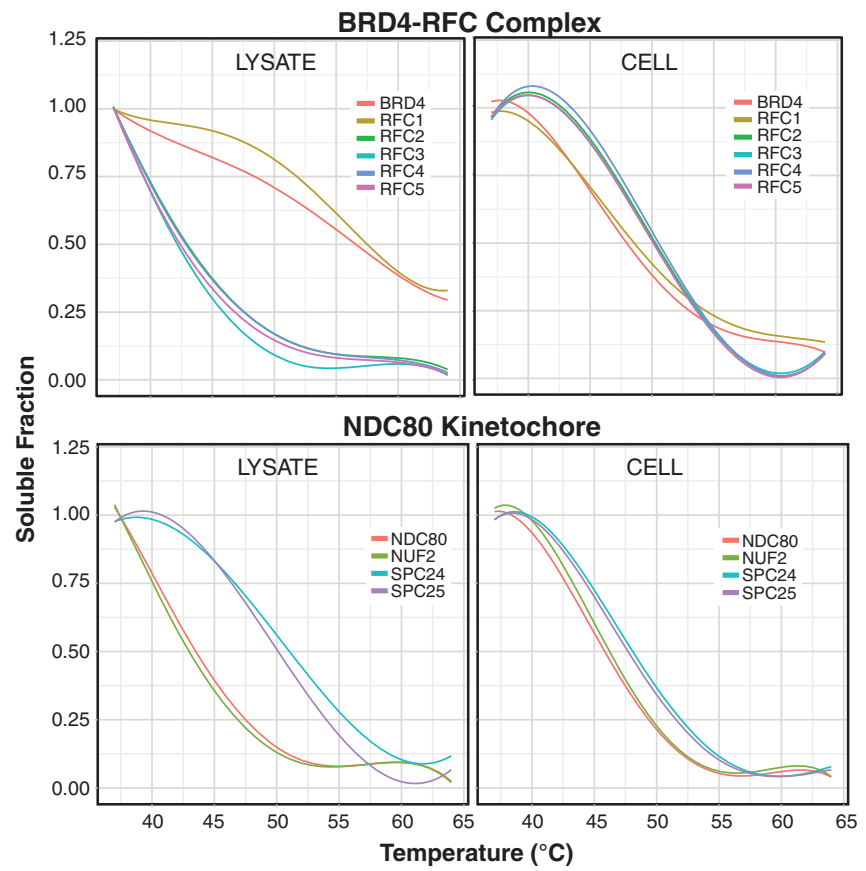

$\mathbf{F}$

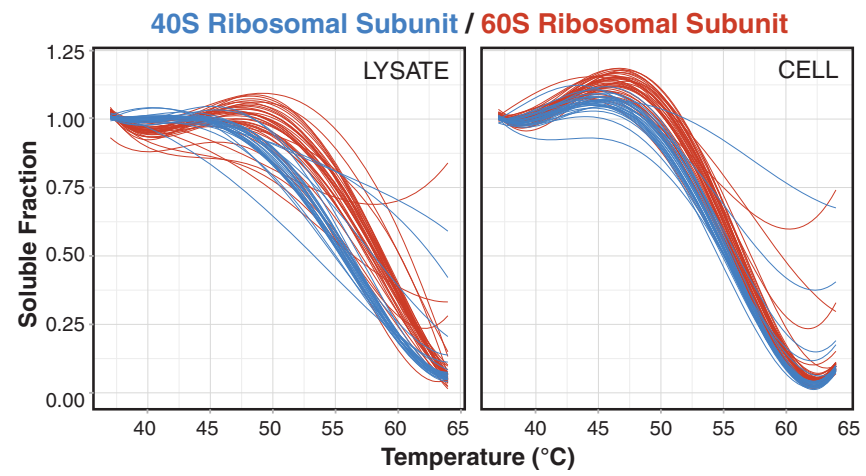

respective protein subset. (C) Number of CORUM protein complexes with nonrandom TPCA behavior at different statistical thresholds. (D) Example of DNA-chromatin- and membrane-associated complexes. (E and $\mathbf{F}$ ) Examples of subcomplexes that differ in cell and in lysate. (G) Schematics of structure for NDC80 kinetochore. 
influence its TPCA signature. We analyzed such data for the interacting protein pairs reported with highest confidence in Hein et al. (9) and observed positive correlation of the TPCA signature with interaction stoichiometry (Spearman's $R=-0.22, P<0.001$, Fig. 3A and fig. S8A) and abundance stoichiometry (Spearman's $R=-0.21$, $P<0.001$, Fig. 3B and fig. S8B). The protein pairs with both interaction stoichiometry and abundance stoichiometry greater than $80 \%$ exhibit much stronger TPCA signatures than those meeting the two criteria separately (Fig. 3C). In comparison, proteins paired solely by similar abundance do not exhibit strong TPCA signatures [fig. S9, A to $\mathrm{C}$, and table S9 (20)]. Accordingly, multiplying interaction stoichiometry with abundance stoichiometry results in values that correlate better with TPCA signature (Spearman's $R=-0.27, P<$ 0.001, Fig. 3D and fig. S8C). Thus, interaction and abundance stoichiometry between interacting proteins are intrinsically captured by TPCA, albeit semiquantitatively.

Under the core-attachment model, subsets of proteins form the stable core of protein complexes that are differentially or temporally bound by other proteins $(21,22)$. We investigated this model with TPCA, comparing melting curves of protein pairs from the complex core to curves for pairs between the core and the attachment subunits (Fig. 3E). We identified the core-core pairs in each complex as those found in two or more other CORUM complexes and core-attachment pairs as those found in that complex only. For most of the complexes, the TPCA signature of core-core pairs is either similar to or much stronger than that of the core-attachment pairs (Fig. 3F). This is in accordance with the core-attachment model in which core-attachment interactions generally occur at lower stoichiometry. Thus, TPCA signature potentially encapsulates the temporal and core-attachment organization of many complexes.

Next, we explored TPCA for system-wide monitoring of protein complex dynamics between cell states. We obtained intact cell MS-CETSA data from K562 cells arrested in the S phase of the cell cycle using methotrexate (fig. S10) and from dimethyl sulfoxide (DMSO)-treated unsynchronized cells (tables S10 and S11). We assessed statistical significance for enhanced TPCA (i.e., higher average curve similarity) observed for any CORUM complex (see materials and methods). We identified 18 protein complexes, some containing overlapping subunits, that have statistically enhanced TPCA signature across both biological replicates in $\mathrm{S}$ phase-synchronized cells (tables S12 to S14).

All but three identified modulated protein complexes had previously been implicated in the S phase. They include the CAF-1 complex (Fig. $3 \mathrm{G}$ ), which forms and localizes to the replication fork during S phase (23); the TREX-THO complex (Fig. 3G, bottom right) required for replication fork progression $(24,25)$; and the TRAP complex required for $\mathrm{S}$ phase progression (26). We also identified many chromatin-nucleosome remodeling and associated complexes such as
BAF (Fig. 3G, top right), LARC, and BRG1-SIN3A (27), consistent with the expected remodeling of chromatin during the $\mathrm{S}$ phase. Histone mRNAs were up-regulated prior to $S$ phase but were rapidly degraded with halted DNA replication (28). We identified three modulated protein complexesthe mRNA decay complex, the exosome, and the integrator complex-that are involved in histone mRNA degradation. Deletion of RRP6, a subunit of exosome, was reported to increase histone mRNA HTB1 during S phase (29), thus highlighting the role of this complex during $\mathrm{S}$ phase. Integrator complex was implicated in the processing of replication-dependent histone mRNAs (30)

Two of the three identified complexes not known to be implicated in S phase are the MDC1-MRNATM-FANCD2 and the DNA ligase III-XRCCPNK-polymerase III complexes. However, they are involved in the DNA damage response in accordance with the known DNA-damaging effect of methotrexate (31). The third complex not implicated in $\mathrm{S}$ phase is the tumor necrosis factor$\alpha$ (TNF- $\alpha$ )-nuclear factor $\kappa \mathrm{B}$ (NF- $\kappa \mathrm{B})$ signaling complex (Fig. 3G). We validated that methotrexate increases the assembly of the associated IкB $\alpha$ p65-p50 protein complex by suppressing activation of the NF-кB signaling pathway, in both the presence and absence of TNF, through inhibiting phosphorylation of the I $\kappa \mathrm{B}$ kinase $\alpha / \beta(\mathrm{IKK} \alpha / \beta)$ complex, IкB $\alpha$, and p65 in K562 cells (Fig. $3, \mathrm{H}$ and I). We subsequently combined data from both replicates-the better data coverage and precision (table S15) allowed us to uncover more complexes that are differentially modulated between $\mathrm{S}$ phase and unsynchronized cells (table S16 and fig. S11).

The enhanced TPCA signature of identified complexes largely arose from curve convergence of most subunits (Fig. 4A). Nevertheless, this could arise from synchronized changes in protein expression. Thus, we quantified relative protein abundance between the DMSO- and methotrexate-treated cells using MS. We observed high reproducibility in relative abundance (Pearson's $R=0.87$, fig. $\mathrm{S} 12$ and table S17) across biological replicates and identified only five subunits $(P<0.05$, or nine at $P<0.1$ ) with differential protein expression (Fig. 4A and table S18) that are mostly parts of the mediator and integrator megacomplexes. Thus, most subunits exhibit enhanced TPCA signature with each other without differential protein expression (Fig. 4A).

Next, we analyzed TPCA signature across multiple cell lines. We generated MS-CETSA data from HEK 293T, A375, HCT116, MCF7, and HL60 intact cells. Combining data from two biological replicates, each with two technical MS runs (average Pearson's $R=0.92$ between biological replicates, fig. S13), we obtained melting curves for $\sim 7600$ proteins on average for each cell line (fig. S14A and tables S19 to S23). On average, $33.8 \%$ of the qualified CORUM complexes exhibit a nonrandom TPCA signature $(P<0.05)$ in each cell line (figs. S14B and S15 to S20), with $~ 70 \%$ overlap between cell lines. We also obtained data from mouse liver and observed $\sim 37.7 \%$ of qualified protein complexes (three or more subunits with melting curves) with nonrandom TPCA signatures (tables S24 to S26 and figs. S21 and S22). Thus, TPCA is observable across multiple cell lines and from tissue samples. Many protein complexes exhibit strong TPCA behavior across the six cell lines (fig. S23), but we also found many complexes with high quality but distinct curves across cell lines (figs. S24 and S25). This suggests plausible variation in protein complex stoichiometry and composition among cell lines, even for fundamental and abundant protein complexes, that could arise from changes in interaction stoichiometry and/ or protein abundance.

Subsequently, we generated weighted networks of reported interactions among proteins identified in all the six cell lines with TPCA-derived $z$-scores (table S26). A basal network averaging the $z$-scores, after removing the highest and the lowest $z$-scores for each interaction, is also constructed. Comparing the basal network with cell-specific TPCA-weighted networks facilitates identifying potentially differentiated interactions, pathways, and functional modules (table S27). Focusing on HCT116, we found that the RAS-RAF-MEK-ERK pathway contains many interactions with highly differentiated TPCA signatures (Fig. 4B). Retrospectively, we observed marked similarity in melting curves of BRAF and RAF1 proteins in HCT116 cells compared to other cell lines (Fig. 4C), consistent with expected dimerization of BRAF and RAF1 driven by active KRAS ${ }^{\mathrm{G} 13 \mathrm{D}}$ in HCT116. TPCA analysis also suggests that BRAFCRAF interaction with MEK is up-regulated in HCT116 cells but is down-regulated in A375 cells, which express $\mathrm{BRAF}^{\mathrm{V} 600 \mathrm{E}}$ (Fig. 4B). This is in line with the recent finding that the interaction is upregulated in cells expressing mutant KRAS and wild-type BRAF but suppressed in cells with mutant BRAF (32). Thus, TPCA could potentially capture cell-specific interactions and pathways.

Lastly, we validated that known and potentially previously unknown protein complexes can be identified from the existing human interactome map using graph or network clustering algorithms $(22,33-36)$ with TPCA-based scoring of interactions (Fig. 4D, figs. S26 and S27, and supplementary text). The best performance was obtained with the $\mathrm{COACH}$ algorithm, which incorporates the core-attachment model of protein complexes (22). Using this algorithm and TPCAbased scoring of interactions gives a performance comparable to that of published interaction reliability scores that incorporate publication count and functional similarity (37). We also observed that TPCA profiling in its current format carries predictive power for protein-protein interactions, with the area under the curve ranging from 0.62 to 0.79 depending on the interaction data sets (Fig. 4, E and F), which collectively suggests that TPCA profiles could also serve to discover new interactions and protein complexes in combination with other approaches.

TPCA enables the intracellular study of the dynamics of multiple protein complexes simultaneously in intact nonengineered cells and tissues. About one-third of the qualified CORUM complexes in each cell line exhibit nonrandom TPCA 

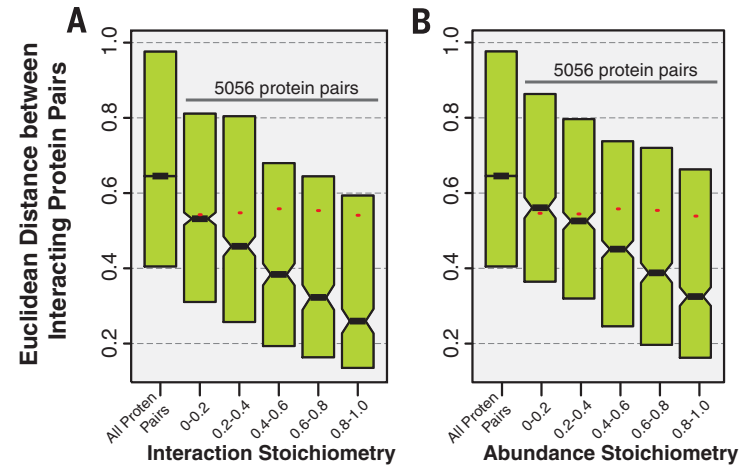

Average Eucl. Distance between
Protein Pairs in Each Complex

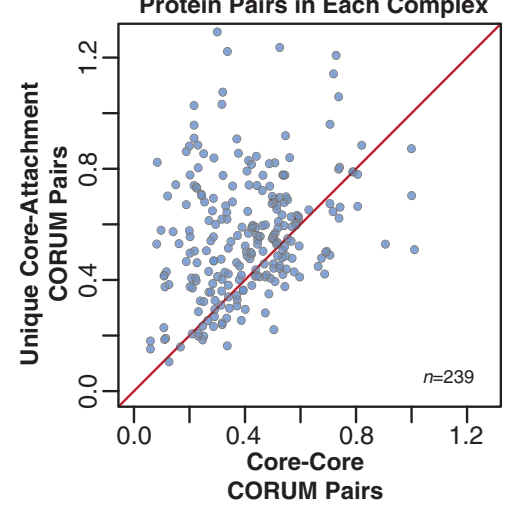

H

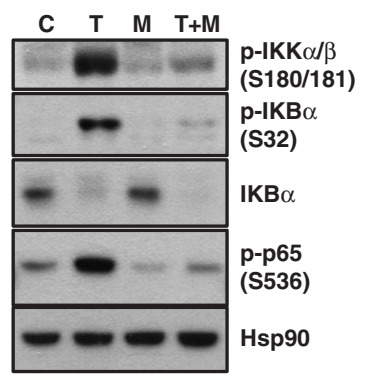

D

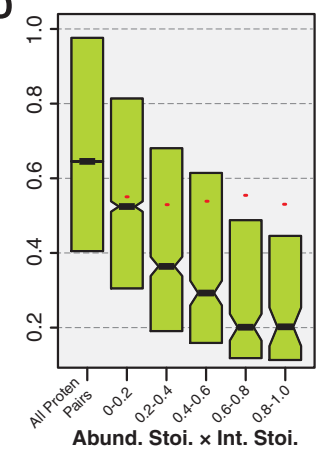

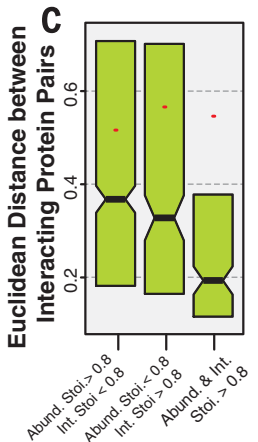

E Core Complex

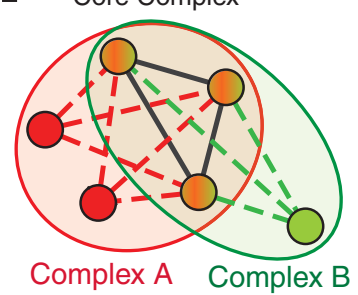

- Non-unique $\mathbf{c}$ - - Unique Core-
G

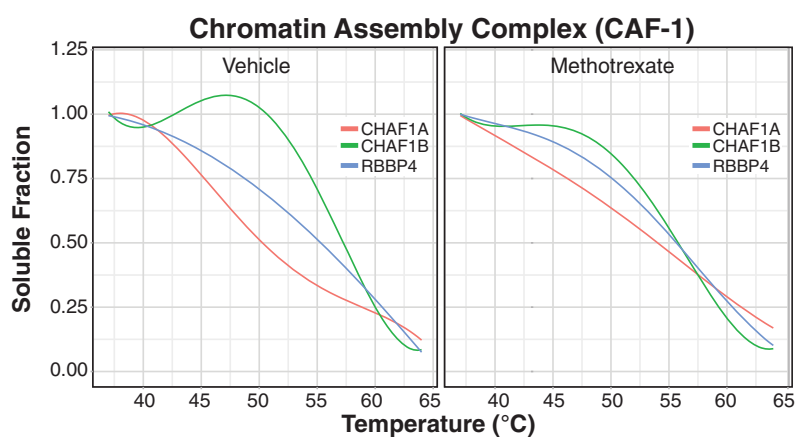

TNF $\alpha / N F K B$ signaling complex

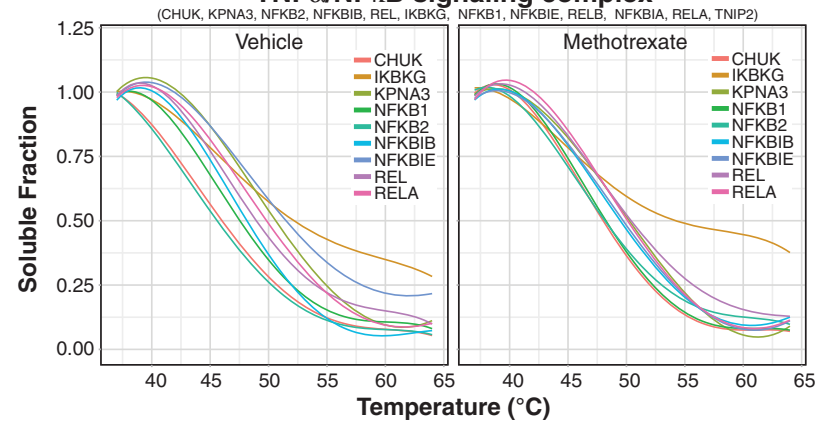

I
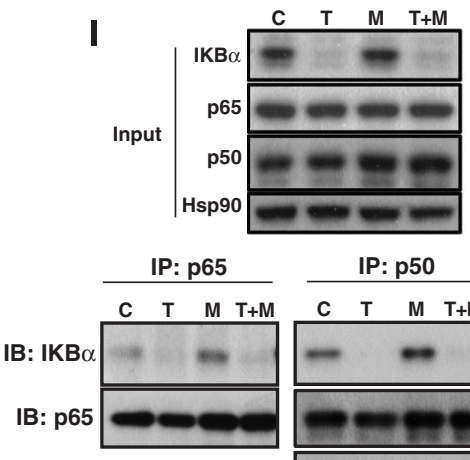

IP: $\lg G$

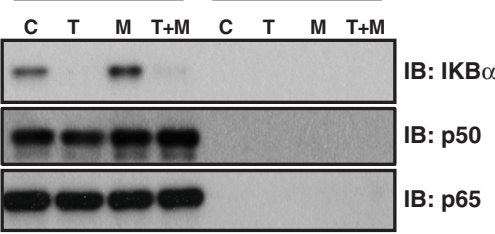

Fig. 3. TPCA signature interaction stoichiometry and abundance stoichiometry between interacting proteins. Correlation of TPCA signature (intact cell MS-CETSA data) with (A) protein interaction stoichiometry and (B) protein abundance stoichiometry of interacting protein pairs reported in Hein et al. (9). (C) Protein pairs with high stoichiometry in both parameters exhibiting the highest TPCA signature. (D) Multiplication of interaction and abundance stoichiometry correlates better with TPCA signature. (E) Coreattachment model for functional organization of protein complexes. (F) Coreattachment protein pairs of a complex generally exhibit weaker TPCA signature than core-core protein pairs. Each dot represents a protein complex.

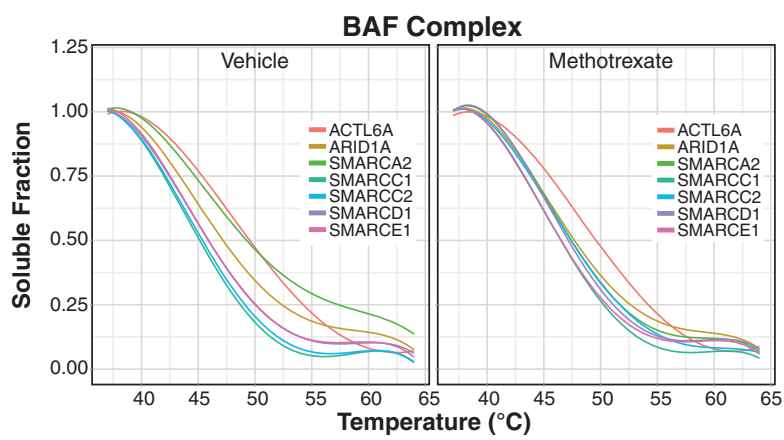

TREX/THO Complex

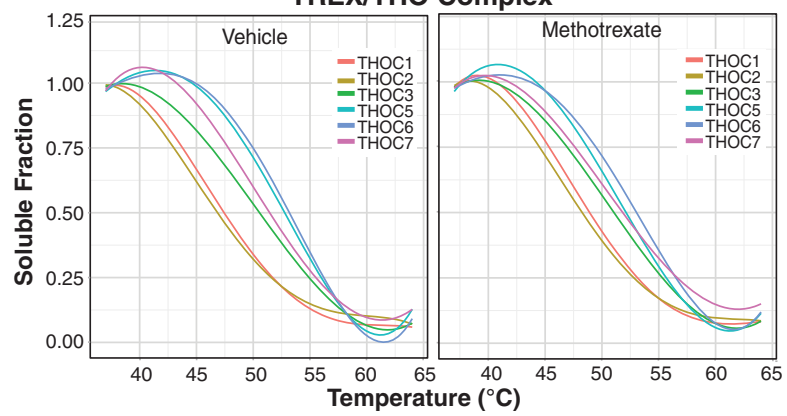

(G) Melting curves for subunits of the chromatin assembly complex 1 (CAF-1), the chromatin-remodeling BAF complex, the NF-kB complex, and the THO complex from methotrexate-treated and vehicle-treated K562 cells.

(H) Assessment of the NF- $\mathrm{kB}$ signaling pathway upon methotrexate treatment in $\mathrm{K} 562$ cells. Western blotting analysis of canonical NF- $\mathrm{KB}$ signaling pathway members in whole-cell lysate. (I) Immunoprecipitation of NF-kB p65 and p50 showing increased interaction with $1 \kappa \mathrm{B} \alpha$. upon methotrexate treatment. C, control (DMSO); T, TNF- $\alpha$; M, methotrexate; T+M, TNF- $\alpha+$ methotrexate. IP, immunoprecipitation; IB, immunoblotting; IgG, immunoglobulin G. Hsp90 served as a loading control. 
A

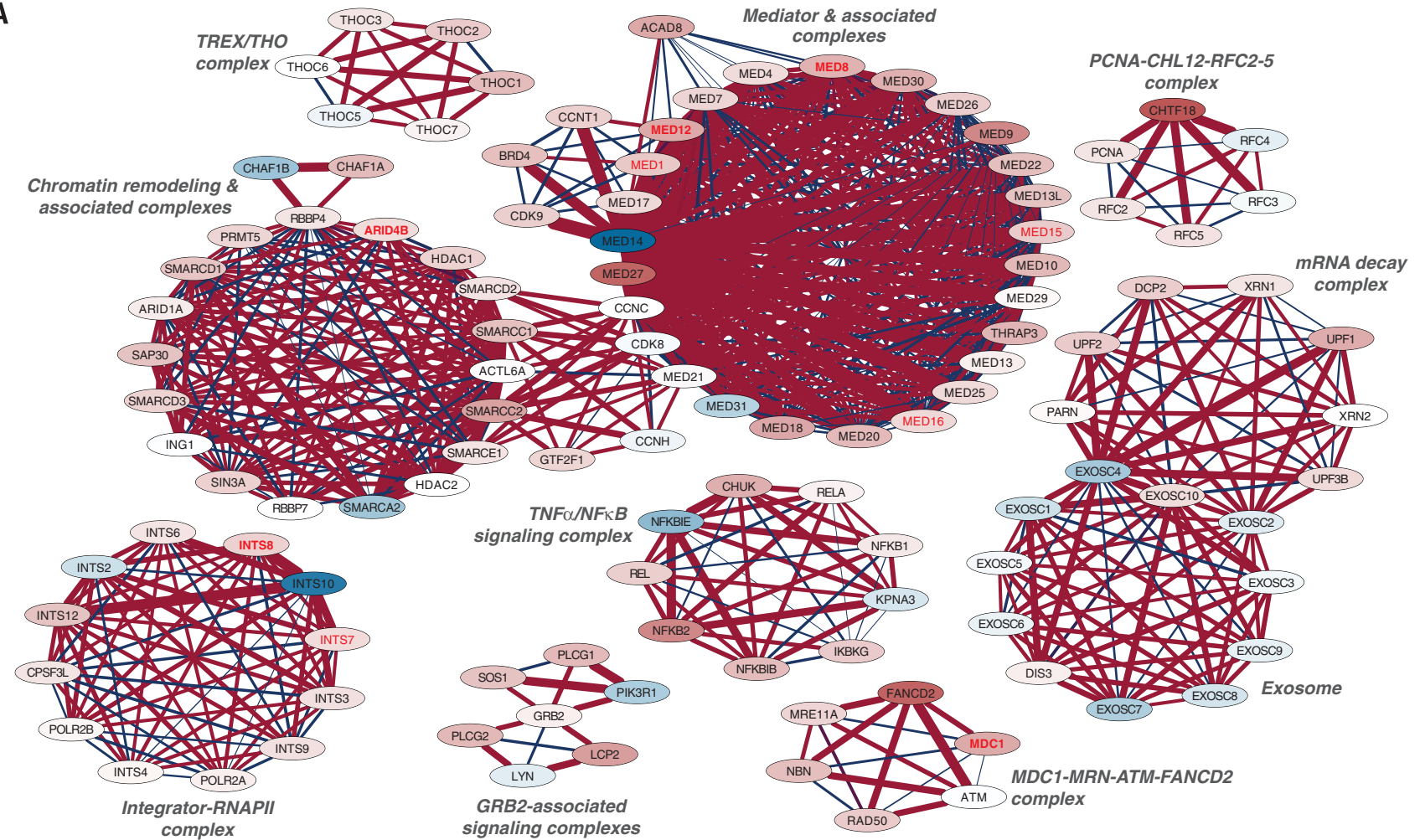

B

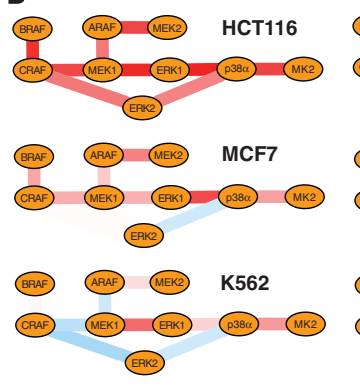

D

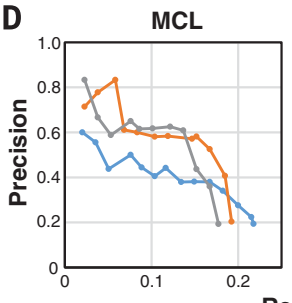

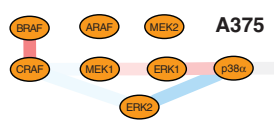

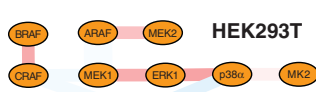
(사우

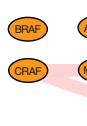

C

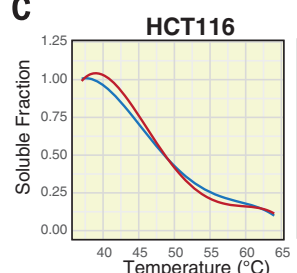
HEK293T

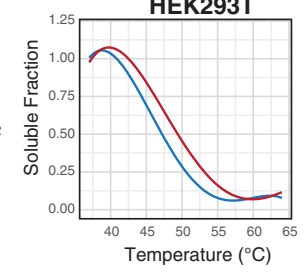

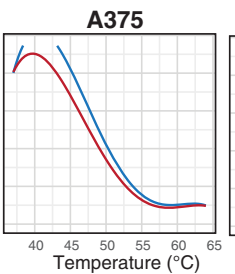
K562

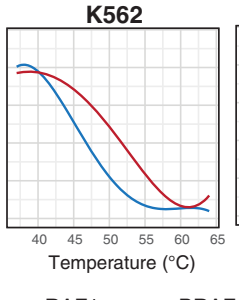

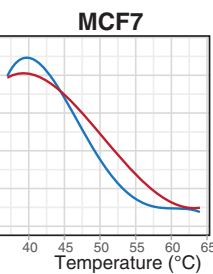
HL60

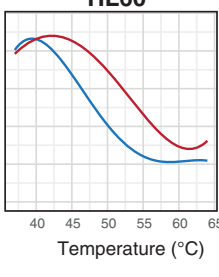

- RAF1 - BRAF

E

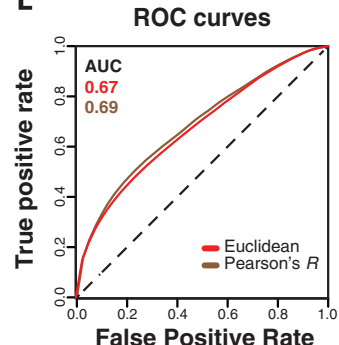

False Positive Rate

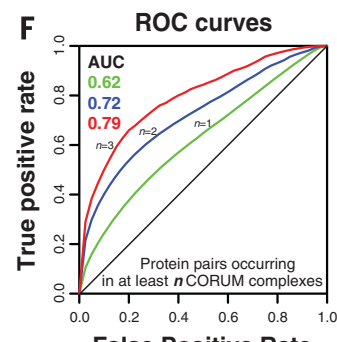

Fig. 4. TPCA signature reveals cell-specific interactions and is predictive of protein complexes. (A) Network view of protein complexes with nonrandom increase in TPCA behavior in methotrexate-treated $\mathrm{K} 562$ cells. Subunits of protein complexes are visualized as nodes with the edge connecting every pair of subunits within a complex. A red node indicates that the protein has greater stability in methotrexate-treated cells than in vehicletreated cells, whereas a blue node indicates the reverse. Color intensity correlates linearly with difference in stability. A red line or edge between two nodes indicates that melting curves of the two proteins are more similar in methotrexate-treated cells, and a blue line or edge indicates the reverse. Line or edge width correlates linearly with difference in melting curve similarity. Differentially expressed proteins are indicated in red $(P<0.10)$ and bold type $(P<0.05)$. (B) TPCA analysis reports differentiated interactions in RAF-MEKERK pathway in HCT116. TPCA-derived $z$-scores are used to facilitate comparison of TPCA signatures across cell lines. A higher $z$-score implies increased interactions. (C) Melting curves of BRAF and RAF1 across the six cell lines. (D) Prediction of CORUM protein complexes using graph or network clustering algorithms on protein interaction network weighted with publication count, reliability score (publication count + function similarity) (33), and TPCA-based scoring. Precision and recall were evaluated against CORUM reference complexes (of size $>3$ ) with Jaccard similarity $\geq 0.5$ considered as match. (E) Predictability of interacting protein pairs (table S2) reported by two or more publications using TPCA. (F) Predictability of co-complex pairs using TPCA. Melting curve data of intact K562 cells (table S7) are used. Euclidean distance between all protein pairs with melting curve is computed and ranked by ascending order (lower Euclidean distance first). All protein pairs are considered as negative data except those known to interact (table S2) or annotated as part of CORUM complexes. 
signatures, with $58 \%$ of the complexes exhibiting nonrandom TPCA signatures in at least one of the cell lines profiled. Membrane-embedded complexes are included in CORUM but are likely not amenable to the protocol adopted in this work (13). Nonsignificant TPCA signatures could arise for protein complexes with very low interaction stoichiometry-increased assembly can be monitored by changes in TPCA signature. TPCA profiling suggests that many complexes can remain intact yet thermally destabilized in the absence of interaction with DNA and LMW ligands such as ATP (Fig. 2, A and E). We observed many mitochondrial proteins that seem more temperatureresistant in intact cells, as observed previously (11). It is unclear whether TPCA is intrinsic and captured by the recent limited proteolysis and MS methodology (38).

We observed many more complexes with significant TPCA signature in cells than in lysates, suggesting that TPCA could potentially aid the intracellular studies of weak or transient proteinprotein interactions that are not preserved in lysate, including protein complexes that depend on the integrity of chromatin-DNA, membrane, and associated structures for stability. TPCA could be used to validate complexes identified by other proteome-wide methodologies and help in their functional characterization across different cell states and conditions. TPCA also permits studying chemical modulators of protein complexes and interactions directly in nonengineered cells and tissues. Analogous to quantifying expression of genes and proteins from a reference genome and reference proteome, respectively, we envisage TPCA-based profiling with reference interactomes as a system-wide discovery strategy for modulated cellular processes. The method therefore can facilitate the discovery of protein complexes involved in diseases, some as potential therapeutic targets or, by TPCA profiles in patient tissue samples, for prognosis of disease progression or optimization of therapy.

\section{REFERENCES AND NOTES}

1. T. Ideker, R. Sharan, Genome Res. 18, 644-652 (2008).

2. X. Wang et al., Nat. Biotechnol. 30, 159-164 (2012).

3. A. Malovannaya et al., Cell 145, 787-799 (2011).

4. P. C. Havugimana et al., Cell 150, 1068-1081 (2012).

5. E. L. Huttlin et al., Cell 162, 425-440 (2015).

6. T. Rolland et al., Cell 159, 1212-1226 (2014).

7. R. M. Ewing et al., Mol. Syst. Biol. 3, 89 (2007)

8. U. Stelzl et al., Cell 122, 957-968 (2005).

9. M. Y. Hein et al., Cell 163, 712-723 (2015).

10. D. Martinez Molina, P. Nordlund, Annu. Rev. Pharmacol. Toxicol. 56, 141-161 (2016)

11. M. M. Savitski et al., Science 346, 1255784 (2014)

12. K. V. Huber et al., Nat. Methods 12, 1055-1057 (2015)

13. F. B. Reinhard et al., Nat. Methods 12, 1129-1131 (2015)

14. A. Chatr-Aryamontri et al., Nucleic Acids Res. 43, D470-D478 (2015)

15. H. Hermjakob et al., Nucleic Acids Res. 32, D452-D455 (2004)

16. L. Licata et al., Nucleic Acids Res. 40, D857-D861 (2012).

17. A. Ruepp et al., Nucleic Acids Res. 38 (suppl. 1), D497-D501 (2010)

18. C. Ciferri et al., Cell 133, 427-439 (2008).

19. H. W. Wang et al., J. Mol. Biol. 383, 894-903 (2008).

20. J. R. Wiśniewski, M. Y. Hein, J. Cox, M. Mann, Mol. Cell. Proteomics 13, 3497-3506 (2014).

21. Z. Dezso, Z. N. Oltvai, A. L. Barabási, Genome Res. 13, 2450-2454 (2003)

22. M. Wu, X. Li, C. K. Kwoh, S. K. Ng, BMC Bioinformatics 10, 169 (2009).

23. M. Hoek, B. Stillman, Proc. Natl. Acad. Sci. U.S.A. 100 12183-12188 (2003).

24. B. Gómez-González, I. Felipe-Abrio, A. Aguilera, Mol. Cell. Biol. 29, 5203-5213 (2009).

25. R. E. Wellinger, F. Prado, A. Aguilera, Mol. Cell. Biol. 26 3327-3334 (2006)

26. M. Ito, C. X. Yuan, H. J. Okano, R. B. Darnell, R. G. Roeder, Mol. Cell 5, 683-693 (2000)

27. H. Cho et al., Mol. Cell. Biol. 18, 5355-5363 (1998).

28. W. F. Marzluff, E. J. Wagner, R. J. Duronio, Nat. Rev. Genet. 9 , 843-854 (2008)

29. R. Canavan, U. Bond, Nucleic Acids Res. 35, 6268-6279 (2007).

30. J. R. Skaar et al., Cell Res. 25, 288-305 (2015).

31. S. A. Martin et al., EMBO Mol. Med. 1, 323-337 (2009).

32. J. R. Haling et al., Cancer Cell 26, 402-413 (2014).

33. G. Liu, L. Wong, H. N. Chua, Bioinformatics 25, 1891-1897 (2009)
34. T. Nepusz, H. Yu, A. Paccanaro, Nat. Methods 9, 471-472 (2012)

35. S. Pu, J. Vlasblom, A. Emili, J. Greenblatt, S. J. Wodak, Proteomics 7. 944-960 (2007).

36. M. Li, J. E. Chen, J. X. Wang, B. Hu, G. Chen, BMC Bioinformatics 9, 398 (2008).

37. H. N. Chua, W. K. Sung, L. Wong, Bioinformatics 22, 1623-1630 (2006).

38. P. Leuenberger et al., Science 355, eaai7825 (2017)

\section{ACKNOWLEDGMENTS}

We thank H. Y. Chang for support and A. Larsson for suggestions. Funding: This research was funded directly by Young Investigator Grant (1610151038) awarded to C.S.H.T. by the Biomedical Research Council of the Agency for Science, Technology and Research (A*STAR). Support for this research was provided by a startup grant from Nanyang Technological University and grants from the Swedish Research Council, the Swedish Cancer Society, and the Knut and Alice Wallenberg foundation awarded to P.N., and by National Medical Research Council (NMRC) grant MOHIAFCAT2/004/2015 to P.N. and R.M.S. Research in the lab of V.T. is supported by grant NRF2016NRF-CRP001-024 from the National Research Foundation Singapore. P.K. and X.B. are supported by the Biomedical Research Council, A*STAR, and NMRC-CBRG14nov086 grants. Authors contributions: P.N. initiated the study; C.S.H.T. conceptualized TPCA and designed and implemented associated algorithms; C.S.H.T, X.B., P.K., V.T. R.M.S., and P.N. designed and supervised experiments; C.S.H.T. and C.H.Y. performed computational analysis; K.D.G., C.S.H.T.,

X.B., M.B.O., L.D., N.P., Y.T.L., and L.S. performed experiments;

R.M.S. and J.L. supervised MS analysis; C.S.H.T. wrote the original manuscript; and C.S.H.T. and P.N. reviewed and edited the

manuscript. Competing interests: P.N. is the inventor of a patent controlled by Pelago Biosciences $A B$ and Evitra Proteoma $A B$ covering the basic CETSA method. J.L. is a paid consultant of Pelago Biosciences AB for MS analysis. All other coauthors declare no competing interests. Data and materials availability: All data are available in the supplementary materials.

\section{SUPPLEMENTARY MATERIALS}

www.sciencemag.org/content/359/6380/1170/suppl/DC1 Materials and Methods

Supplementary Text

Figs. S1 to S27

Tables S1 to S27

22 February 2017; resubmitted 28 September 2017

Accepted 27 January 2018

Published online 8 February 2018

10.1126/science.aan0346 


\section{Science}

\section{Thermal proximity coaggregation for system-wide profiling of protein complex dynamics in cells}

Chris Soon Heng Tan, Ka Diam Go, Xavier Bisteau, Lingyun Dai, Chern Han Yong, Nayana Prabhu, Mert Burak Ozturk, Yan Ting Lim, Lekshmy Sreekumar, Johan Lengqvist, Vinay Tergaonkar, Philipp Kaldis, Radoslaw M. Sobota and Pär Nordlund

Science 359 (6380), 1170-1177.

DOI: 10.1126/science.aan0346originally published online February 8, 2018

\section{Taking the heat together}

Many of the processes in living cells are mediated by protein complexes that dynamically assemble and dissociate depending on cellular needs. Tan et al. developed a method called thermal proximity coaggregation (TPCA) to monitor the dynamics of native protein complexes inside cells (see the Perspective by Li et al.). The method is based on the idea that proteins within a complex will coaggregate upon heat denaturation. It uses a previously described cellular shift assay to determine melting curves for thousands of proteins and assigns a TPCA signature on the basis of similarity between the curves. The method was validated by detection of many known protein complexes. It identified cell-specific interactions in six cell lines, highlighting the potential for identifying protein complexes that are modulated by disease. Science, this issue p. 1170; see also p. 1105

ARTICLE TOOLS

SUPPLEMENTARY MATERIALS

RELATED CONTENT

REFERENCES

PERMISSIONS http://science.sciencemag.org/content/359/6380/1170

http://science.sciencemag.org/content/suppl/2018/02/07/science.aan0346.DC1

http://science.sciencemag.org/content/sci/359/6380/1105.full

This article cites 38 articles, 11 of which you can access for free http://science.sciencemag.org/content/359/6380/1170\#BIBL

http://www.sciencemag.org/help/reprints-and-permissions

Use of this article is subject to the Terms of Service

Science (print ISSN 0036-8075; online ISSN 1095-9203) is published by the American Association for the Advancement of Science, 1200 New York Avenue NW, Washington, DC 20005. The title Science is a registered trademark of AAAS.

Copyright (C) 2018 The Authors, some rights reserved; exclusive licensee American Association for the Advancement of Science. No claim to original U.S. Government Works 